\title{
Quantitative SERS sensors for environmental analysis of naphthalene
}

\author{
O. Péron, ${ }^{a b}$ E. Rinnert, ${ }^{* a}$ T. Toury ${ }^{b}$ M. Lamy de la Chapelle ${ }^{c}$ and C. Compère ${ }^{a}$ \\ Received 14th October 2010, Accepted 24th November 2010 \\ DOI: 10.1039/c0an00797h
}

In the investigation of chemical pollutants, such as PAHs (Polycyclic Aromatic Hydrocarbons) at low concentration in aqueous medium, Surface-Enhanced Raman Scattering (SERS) stands for an alternative to the inherent low cross-section of normal Raman scattering. Indeed, SERS is a very sensitive spectroscopic technique due to the excitation of the surface plasmon modes of the nanostructured metallic film. The surface of quartz substrates was coated with a hydrophobic film obtained by silanization and subsequently reacted with polystyrene (PS) beads coated with gold nanoparticles. The hydrophobic surface of the SERS substrates pre-concentrates non-polar molecules such as naphthalene. Under laser excitation, the SERS-active substrates allow the detection and the identification of the target molecules localized close to the gold nanoparticles. The morphology of the SERS substrates based on polystyrene beads surrounded by gold nanoparticles was characterized by scanning electron microscopy (SEM). Furthermore, the Raman fingerprint of the polystyrene stands for an internal spectral reference. To this extent, an innovative method to detect and to quantify organic molecules, as naphthalene in the range of 1 to $20 \mathrm{ppm}$, in aqueous media was carried out. Such SERSactive substrates tend towards an application as quantitative SERS sensors for the environmental analysis of naphthalene.

\section{Introduction}

High quality factors of surface plasmon modes lead SurfaceEnhanced Raman Scattering (SERS) as a very sensitive spectroscopic technique. Huge Raman enhancements, up to $10^{14}$ fold $^{1-5}$ the normal Raman signal of non-adsorbed molecules, have been observed when molecules are adsorbed near metallic surfaces. Latter, as silver, gold, copper, exhibit high optical reflectivity. ${ }^{6}$ The short-range chemical model and the long-range electromagnetic model ${ }^{7-10}$ help to reveal the Raman enhancement origin. The first one is related to a charge transfer between the roughened surface and the target molecule via an increase in adsorbed molecule polarisability. The second one is linked to the excitation of surface plasmons leading to an increase in the local electromagnetic field. A final Raman enhancement of an adsorbed molecule can be considered as the coupling of both mechanisms.

Many studies were realized on polystyrene (PS) beads chemically modified for the purpose of grafting metal nanoparticles. Functionalization of the polystyrene beads can occur thanks to

${ }^{a}$ IFREMER, Service Interfaces et Capteurs, Département Recherches et Développements Technologiques, BP70, 29280 Plouzané, France. E-mail: Emmanuel.Rinnert@ifremer.fr; Fax: +33 (0)2-98-22-45-35; Tel: +33 (0)2-98-22-41-61

${ }^{b} I C D$ - LNIO - UMR CNRS 6279, Université de Technologie de Troyes, 12 rue Marie Curie, 10010 Troyes, France

${ }^{c}$ Laboratoire CSPBAT (FRE 3043), UFR SMBH, Université Paris XIII, 74 rue Marcel Cachin, 93017 Bobigny, France a modification of the surface electric charge,${ }^{11-13}$ a thiolation ${ }^{14}$ or an amination ${ }^{15}$ of the PS beads. In both latter cases, the functional groups $-\mathrm{SH}$ or $-\mathrm{NH}_{2}$ have an affinity for metallic particles: gold is bound to the surface through covalent interactions. ${ }^{16-18}$ The fabrication of SERS-active substrates through PS beads coated with metal nanoparticles has been lately employed in a wide variety of applications. For example, the crystal violet dye as a probe has been used, ${ }^{19}$ the identification of benzenethiol adsorbed on $\mathrm{Ag}$ films, ${ }^{20}$ the detection of streptavidin molecules after biotin grafting ${ }^{21}$ and the investigation for chemical components in saliva. ${ }^{22}$ In our case, a facile method based on Debye adsorption - the interaction between a permanent dipole and an induced dipole - is employed to coat non-polar PS beads with gold nanoparticles. Although silver presents a higher SERS response with an enhancement factor 10-100-fold greater than gold, $\mathrm{Au}$ was chosen here on account of $\mathrm{AgCl}$ formation on silver surfaces in the marine medium. ${ }^{23}$ Such a facile method used in core-shell fabrication ${ }^{24}$ has been chosen here in order to avoid the addition of organic matter with terminal functional groups whose Raman fingerprints may represent a drawback for the SERS spectra exploitation. Subsequently, PS beads decorated with gold nanoparticles were immobilized onto a quartz substrate previously functionalized with (3-mercaptopropyl)trimethoxysilane (MPTMS). The MPTMS film and the PS beads stand for the hydrophobic part allowing the pre-concentration of apolar molecules such as PAHs (Polycyclic Aromatic Hydrocarbons).

Herein the aim of the study is to give an innovative method to detect and to quantify organic molecules as PAHs at low 
concentration in aqueous media. Such target molecules are of interest for the exploitation and exploration of oceanic and energetic resources. In addition, the detection of PAHs, such as naphthalene, which is a chemical pollutant, presents a societal interest (DCE Directive Cadre sur l'Eau 200/60/CE). Nowadays, there is a lack of a relevant technique that allows qualitative, quantitative, fast and in situ analysis of PAHs in aqueous media.

The low solubility of PAHs in the marine medium requires the development of sensitive substrates. Hydrophobic SERS-active substrates offer the expected solution. ${ }^{25-29}$ Several papers present the SERS effect as a quantitative analytical tool using: the quantitative control of the substrate-withdrawal method onto adsorbed sodium benzoate, ${ }^{30}$ the partial least-squares (PLS) algorithm to determine glucose detection, ${ }^{31}$ self-assembled monolayers (SAMs) as an internal standards and PLS, ${ }^{32}$ isotopically labeled creatinine as internal standard and PLS, ${ }^{33}$ nicotine and pyridine respective deuterated isotopomers as internal standards in a microfluidic system. ${ }^{34}$ To the best of our knowledge, this paper reports for the first time SERS measurements using the Raman fingerprint of the polystyrene as an internal spectral reference with a view to quantitative analyses. Naphthalene solutions with concentration in a range of 1-20 ppm have been tested.

\section{Experimental}

\section{Instrumentation}

SERS spectra of naphthalene solutions were recorded at room temperature with a Raman spectrometer (Labram HR800 Horiba Jobin Yvon) using the $632.8 \mathrm{~nm}$ excitation line of a $\mathrm{He}-\mathrm{Ne}$ laser (power $10 \mu \mathrm{W}$ at the sample) in backscattering geometry. The laser beam was focused through a water immersion lens $(\times 100, \mathrm{NA}=1)$ to a spot of around $1 \mu \mathrm{m}^{2}$. Scattered radiation was collected at $180^{\circ}$ relative to the excitation beam and detected with an Andor CCD cooled by the Peltier effect. A 300 g. $\mathrm{mm}^{-1}$ grating coupled to an $800 \mathrm{~mm}$ spectrograph allows a spectral resolution of around $2 \mathrm{~cm}^{-1}$. Spectral calibration was performed on silicon sample (at $520 \mathrm{~cm}^{-1}$ ). Two stage edge filters allow Raman Stokes studies. The accumulation times were $2 \times 10 \mathrm{~s}$.

Scanning Electron Microscopy (SEM) measurements were done using a field emission scanning electron microscope (FEI-Quanta200). Before loading into the observation chamber, the samples were coated with a thin Au film $(\sim 10-15 \mathrm{~nm})$ by sputtering to avoid a surface charging effect.

\section{Chemicals and materials}

$\mathrm{HAuCl}_{4} \cdot 3 \mathrm{H}_{2} \mathrm{O}$ was purchased from Acros Organics. Polystyrene (PS) beads with $800 \mathrm{~nm}$ diameter $( \pm 50 \mathrm{~nm})$, hydrochloric acid (37\%) and (3-mercaptopropyl)trimethoxysilane (MPTMS, 95\%) were obtained from Sigma-Aldrich. Trisodium citrate was purchased from Prolabo, nitric acid (65\%) was from Merck, hydrogen peroxide $(30 \%)$ was from J.T. Baker, ammonium hydroxide $(33 \%)$ was from Riedel-de-Haën, methanol and ethanol were of analytical grade from Fisher. Naphthalene from Fisher was used as the analytical molecular reference.

The quartz substrates were tailored with $8 \mathrm{~mm}$ diameter and $0.8 \mathrm{~mm}$ thickness from Heraeus. The quartz was cleaned using aqua regia $\left(25 \% \mathrm{HCl}, 25 \% \mathrm{HNO}_{3}, 50 \%\right.$ distilled water v/v) and afterwards a base 'piranha' solution $\left(75 \% \mathrm{NH}_{4} \mathrm{OH}, 25 \% \mathrm{H}_{2} \mathrm{O}_{2}\right.$ $\mathrm{v} / \mathrm{v}$ ) to eliminate organic compounds and to promote hydroxylated surfaces.

\section{Polystyrene beads coated with gold nanoparticles and naphthalene solutions}

The solution of PS beads coated with gold nanoparticles was prepared taking inspiration from the method described by Frens. ${ }^{35} 20 \mu \mathrm{L}$ of PS beads of the commercial solution $\left(3.6 \times 10^{11}\right.$ particles per milliliter) dispersed in $5 \mathrm{~mL}$ of distilled water were added to $5 \mathrm{~mL}$ of an ethanolic solution of tetrachloroauric acid at $2 \times 10^{-3} \mathrm{M}$ under stirring. The reactor was placed on a hotplate and magnetically stirred. Temperature mixture was controlled with a thermometer $\left(\sim 90^{\circ} \mathrm{C}\right)$. Then, $10 \mathrm{~mL}$ of sodium tricitrate at $4 \times 10^{-3} \mathrm{M}$ was slowly added. After the addition of the reducing agent, the gold particles start to form during a process known as nucleation and subsequently grow. The initially dark-grey solution turned to a red-violet hue after thirty minutes of continued boiling. After one hour of boiling, the mixture was cooled at room temperature under stirring.

The naphthalene solution was prepared by dissolving PAH in methanol, wherein it is more soluble, and diluted in distilled water. Naphthalene in pure water at $25^{\circ} \mathrm{C}$ is soluble at $31.8 \mathrm{mg}$ $\mathrm{L}^{-1}$. A $20 \mathrm{ppm}$ naphthalene solution was prepared from $62 \mu \mathrm{L}$ of $0.05 \mathrm{~mol} \mathrm{~L}^{-1}$ naphthalene methanol solution and the corresponding blank composed of $62 \mu \mathrm{L}$ of methanol completed by 20 $\mathrm{mL}$ of distilled water.

\section{SERS substrates}

The strategy for obtaining SERS-active substrates was to graft silanes with terminal - SH groups. The silanes are bound chemically to the substrate via hydroxyl groups existing at the quartz surface. ${ }^{36}$ With MPTMS silanization, the quartz surface properties are modified and the non-polar $-\mathrm{CH}_{2}$ groups give hydrophobic surfaces. The non-polar PS beads decorated with gold nanoparticles were strongly grafted to the functionalized quartz surface via covalent bonds between $-\mathrm{SH}$ and Au. ${ }^{16}$

Clean disk quartz substrates were immersed in a $5 \%(\mathrm{v} / \mathrm{v})$ methanol solution of MPTMS for four hours according to the results obtained by Seitz et al. ${ }^{37}$ After silanization, the substrates were rinsed several times in methanol, sonicated in methanol for twenty minutes to remove excess silane and dried for one hour at $100{ }^{\circ} \mathrm{C}$ in an air oven. Then, the silanized quartz were immersed overnight in the gold coated PS bead solution. Finally, SERS substrates were dried for ten minutes at $100{ }^{\circ} \mathrm{C}$. Suitable substrates for adsorbed hydrophobic molecule species and SERS measurements were thus synthesized. Fig. 1 presents a schematic representation of the SERS substrate fabrication procedure.

\section{Results and discussion}

Within the scope of characterizing the morphology of the SERS substrates, Scanning Electron Microscopy (SEM) measurements were carried out. Fig. 2(a) shows an overview of the SERS substrate surfaces. The PS beads, with a measured diameter of around $800 \mathrm{~nm}$, are deployed onto the substrate surface as a monolayer. The SEM images clearly show that the PS bead surfaces are partially coated; gold nanoparticles were locally 
a)
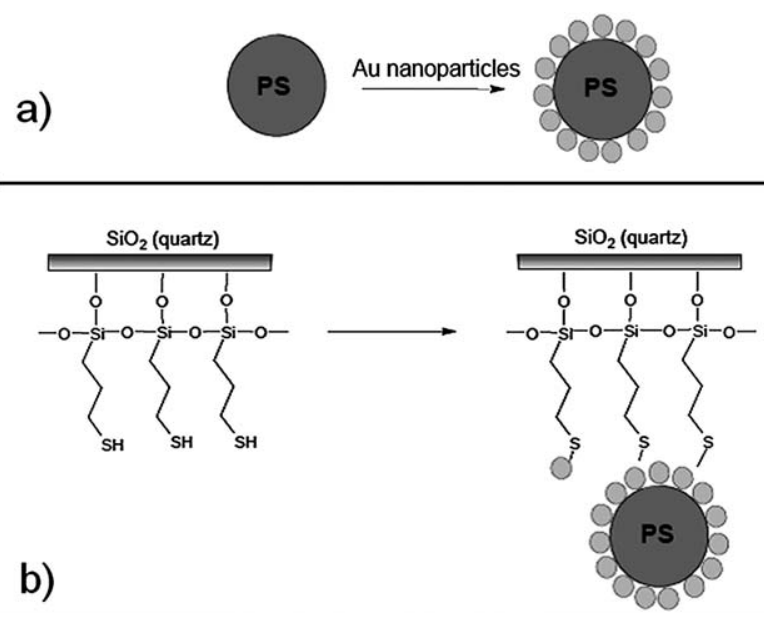

Fig. 1 (a) Schematic of polystyrene beads coated with gold nanoparticles. (b) Schematic of the SERS substrate procedure fabrication: silanization step and after immersion into the gold coated PS bead solution. The scale is not respected.
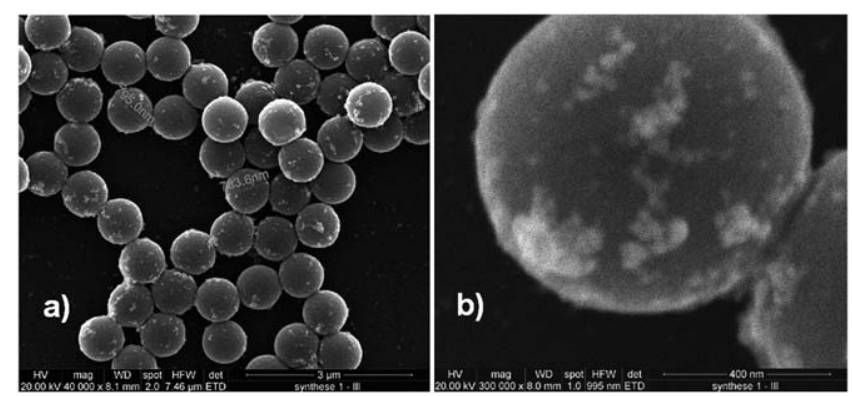

Fig. 2 SEM images of SERS substrate based on polystyrene beads coated with gold nanoparticles (a) $\times 40000$ and (b) $\times 300000$.

grafted to the PS bead surface. Besides gold nanoparticles are also present on the MPTMS. Gold nanoparticles have an estimated diameter of $\sim 50 \mathrm{~nm}$ (Fig. 2(b)). Nevertheless, such a gold coating of PS beads is sufficient to generate the SERS effect. In order to limit the contribution of the SERS effect due to the gold nanoparticles localized onto the MPTMS, all the SERS measurements were performed with the laser beam focused onto the PS beads.

Fig. 3(a) presents the SERS spectrum of the PS beads through the blank and the Raman spectrum of PS solid for comparison. The Raman peaks of the PS solid are almost all present in the SERS spectrum of the PS beads through the blank. The unassigned peaks, in particular in the region $1500-1700 \mathrm{~cm}^{-1}$, can correspond to the MPTMS organic part and the $\mathrm{H}-\mathrm{O}-\mathrm{H}$ bending $\left(1600-1630 \mathrm{~cm}^{-1}\right)$ of water. ${ }^{38}$ The main Raman peak of polystyrene $\left(1004 \mathrm{~cm}^{-1}\right)$ was identified as the ring vibration. ${ }^{39}$ It was located with an arrow in the figure and used as an internal spectral reference in the following quantitative study.

Fig. 3(b) shows the SERS spectra of naphthalene solutions tested by increasing concentration from 1 to $20 \mathrm{ppm}$. Hydrophobic molecule pre-concentrations on non-polar surfaces result in a physisorption process. Physical adsorption is a type of adsorption in whereby the adsorbate is weakly linked to the surface only through van der Waals interactions. More precisely
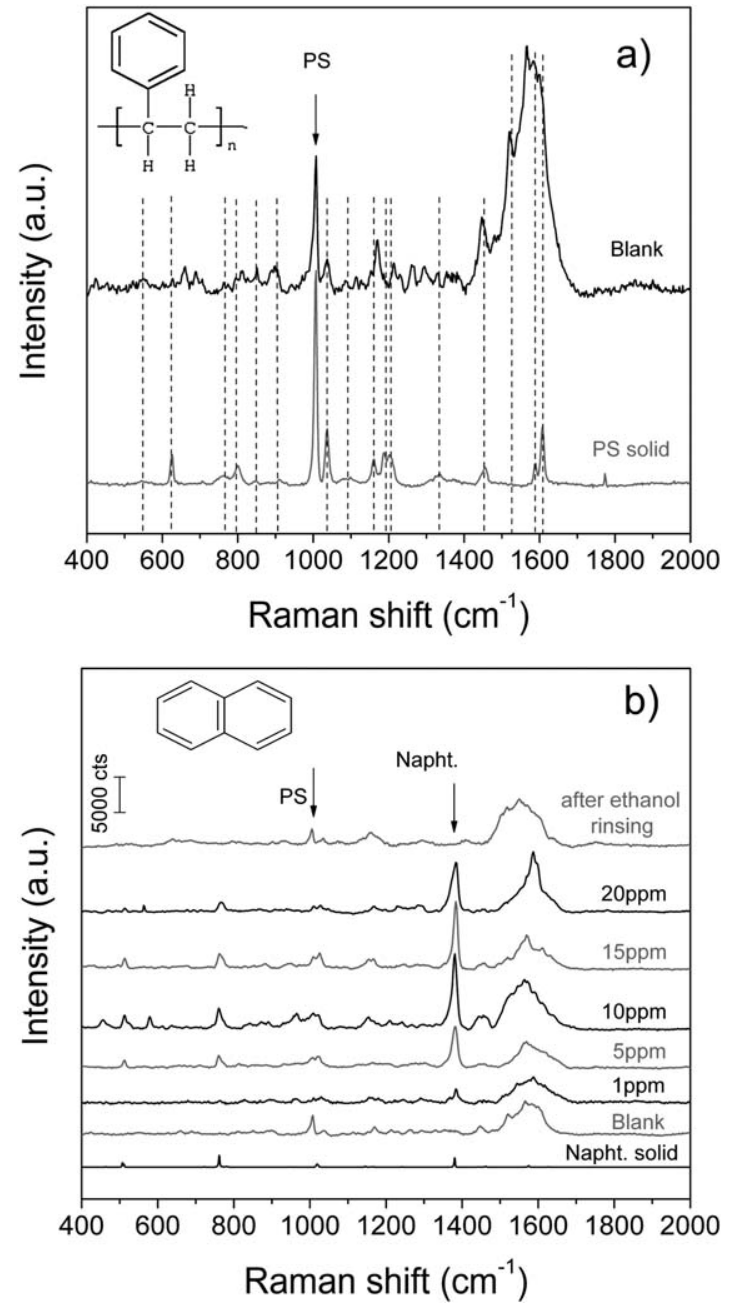

Fig. 3 (a) SERS spectrum of the PS beads through the blank and Raman spectrum of PS solid for comparison. (b) SERS spectra of naphthalene solutions from 1 to $20 \mathrm{ppm}$, PS beads through the blank, PS beads through the blank after ethanol rinsing and for comparison the Raman spectrum of solid-state naphthalene is also given. Integration time of $2 \times 10 \mathrm{~s}$.

here, London dispersion forces arise between non-polar molecules. Rinsing in $10 \mathrm{~mL}$ of ethanol for $10 \mathrm{~min}$ is required to eliminate naphthalene molecules. The results demonstrate that the SERS intensity cannot be used directly with the view to quantify the detection of naphthalene traces. We can not draw any conclusions on the evolution of the spectral intensity of the Raman peaks versus PAH concentration insofar as the isolated sites on the surface, called hot spots, give rise to intense local plasmon fields. ${ }^{40-43}$ Nonetheless, SERS-active substrates based on polystyrene beads surrounded by gold nanoparticles, using the main PS Raman peak as an internal spectral reference, tend towards a quantitative tool (Fig. 4).

Concerning the quantitative proof, Fig. 4 represents the normalized intensity $\left(I_{\mathrm{Napht}} / I_{\mathrm{PS}}\right)$ with regard to the naphthalene concentration in the range of $1-20 \mathrm{ppm}$. The normalized intensity $\left(I_{\mathrm{Napht}} / I_{\mathrm{PS}}\right)$ is the ratio between the band intensity of the naphthalene at $1380 \mathrm{~cm}^{-1}$ and the intensity of the internal spectral reference $\left(1004 \mathrm{~cm}^{-1}\right)$. In the range of 1-20 ppm naphthalene concentration, a linear fit was plotted. Nevertheless, 


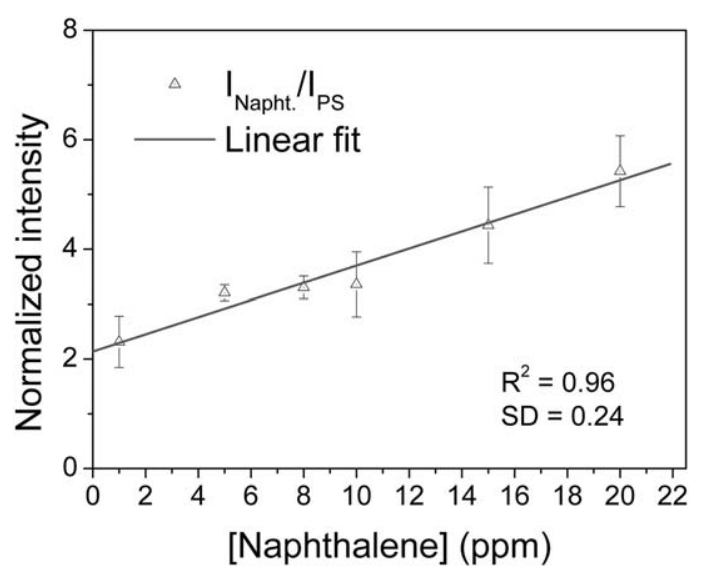

Fig. 4 Normalized intensity $\left(I_{\mathrm{Napht}} / I_{\mathrm{PS}}\right)$ versus the naphthalene concentration in the range $1-20 \mathrm{ppm}$ and the linear fit. Each white triangle corresponds to the mean of 5 measurements at the same concentration. The uncertainty is calculated as the standard deviation.

adsorption isotherm equations ${ }^{44,45}$ could be used to describe the adsorption between the solid phase and aqueous phase at equilibrium. A good agreement is reached with the $R^{2}$ coefficient $\left(R^{2}=0.96\right)$ and the standard deviation of the estimated intensity is $\mathrm{SD}=0.24$. An experimental limit of quantification of $1 \mathrm{ppm}$ was obtained.

The reciprocal fit allowing the use of the sensor in real conditions of application was plotted (not presented here). For example, a solution with an unknown naphthalene concentration can be determined with an uncertainty of $30 \%$ insofar as the naphthalene concentration is $12 \mathrm{ppm}$, i.e. an uncertainty of \pm 3.6 ppm.

For the purposes of studying the repeatability of SERS detection of naphthalene, Fig. 5 gathers the normalized intensity

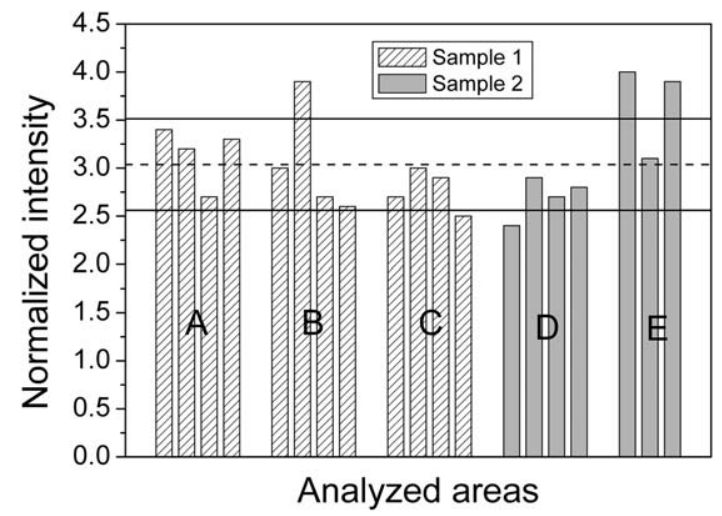

Fig. 5 Histogram of the normalized intensity $\left(I_{\mathrm{Napht}} / I_{\mathrm{PS}}\right)$ of a $10 \mathrm{ppm}$ naphthalene solution versus the different analysed areas (from A to E) of the samples. Each rectangle of the histogram represents an experimental SERS measurement. The samples 1 and 2 refer to two distinct synthesizes of SERS substrates based on the same procedure fabrication. The dashed line corresponds to the mean of all the experimental measurements; the top and bottom black lines are the standard deviation calculated of all the plotted measurements $\mathrm{SD}=0.47(\mathrm{ca} .16 \%)$. The sensor surface was rinsed in $10 \mathrm{~mL}$ ethanol for ten minutes between each tested area.
$\left(I_{\mathrm{Napht}} / I_{\mathrm{PS}}\right)$ of a $10 \mathrm{ppm}$ naphthalene solution with regard to the different analysed areas (from A to E) of the samples.

As a result, the SERS response of the sensor was quite repeatable $(\mathrm{SD}=0.47 ; \mathrm{ca} .16 \%$ ) in the naphthalene detection whatever the tested area of the sample and synthesis to synthesis. To this extent, the repeatability of the experimental measurements with the SERS substrate based on gold coated PS beads was proved. In addition, Fig. 5 displays that 11 rinsing steps with ethanol and water (one step between each spectrum) on sample 1 and 6 rinsing steps on sample 2 do not make the sensor worse according to the homogeneity of the response.

\section{Conclusion}

SERS-active substrates based on PS beads coated with gold nanoparticles were achieved with success. The morphology of the SERS sensors was described as a monolayer of PS beads partially coated with gold nanoparticles. The originality and the advantage of these SERS-active substrates reside in the pre-concentration of naphthalene onto a hydrophobic surface coupled to the use of an internal spectral reference via the Raman fingerprint of the PS beads. This study suggests that a determination of the concentration of a naphthalene solution was feasible in the range of 1-20 ppm with a high repeatability. Such SERS-active substrates tend towards an application as quantitative SERS sensors for environmental analysis. In the near future, in situ quantitative SERS measurements can be planned on hydrophobic molecules of environmental interest such as PAHs.

\section{Acknowledgements}

This project was supported by ANR financing (P2IC: Discomar) and carried out in conjunction with the Universite de Technologie de Troyes (UTT).

The authors acknowledge Philippe Crassous, IFREMER Laboratoire Environnement Profond at Ifremer Plouzané France, for the SEM images.

\section{References}

1 M. Fleischmann, P. J. Hendra, A. J. McQuillan, R. L. Paul and E. S. Reide, J. Raman Spectrosc., 1976, 4, 269-274.

2 D. J. Jeanmaire and R. P. Van Duyne, J. Electroanal. Chem., 1977, 84, $1-20$.

3 M. G. Albrecht and J. A. Creighton, J. Am. Chem. Soc., 1977, 99, 5215-5217.

4 S. Nie and S. R. Emory, Science, 1997, 275, 1102-1106.

5 K. Kneipp, Y. Wang, H. Kneipp, L. T. Perelman, I. Itzkan, R. R. Dasari and M. S. Feld, Phys. Rev. Lett., 1997, 78, 1667-1670.

6 M. Muniz-Miranda, B. Pergolese, A. Bigotto, A. Giusti and M. Innocenti, Mater. Sci. Eng., C, 2007, 27, 1295-1299.

7 M. Moskovits, Rev. Mod. Phys., 1985, 57, 783-826.

8 A. Otto, J. Raman Spectrosc., 1991, 22, 743-752.

9 A. Otto, I. Mrozek, H. Grabhorn and W. Akemann, J. Phys.: Condens. Matter, 1992, 4, 1143-1212.

10 A. W. Wokaun, Solid State Physics, ed. H. Ehrenreich, F. Seitz and D Turnbull, Academic, New York, 1984, vol. 38, p. 223.

11 Y.-C. Cao, X.-F. Hua, Z. Wang, Z.-L. Huang, Y.-D. Zhao, H. Cheng and M.-X. Liu, J. Immunol. Methods, 2006, 317, 163-170.

12 J.-H. Lee, D. O. Kim, G.-S. Song, Y. Lee, S.-B. Jung and J.-D. Nam, Macromol. Rapid Commun., 2007, 28, 634-640.

13 Y.-C. Cao, Z. Wang, X. Jin, X.-F. Hua, M.-X. Liu and Y.-D. Zhao, Colloids Surf., A, 2009, 334, 53-58.

14 K.-T. Yong, Y. Sahoo, M. T. Swihart and P. N. Prasad, Colloids Surf., A, 2006, 290, 89-105. 
15 N. L. Lala, T. C. Deivaraj and J. Y. Lee, Colloids Surf., A, 2005, 269, $119-124$.

16 R. G. Nuzzo, B. R. Zegarski and L. H. Dubois, J. Am. Chem. Soc., 1987, 109, 733-740.

17 K. C. Grabar, K. J. Allison, B. E. Baker, R. M. Bright, K. R. Brown R. G. Freeman, A. P. Fox, C. D. Keating, M. D. Musick and M. J. Natan, Langmuir, 1996, 12, 2353-2361.

18 C. D. Keating, M. D. Musick, M. H. Keefe and M. J. Natan, J. Chem. Educ., 1999, 76, 949-955.

19 S. Nath, S. K. Ghosh, S. Kundu, S. Praharaj, S. Panigraphi, S. Basu and T. Pal, Mater. Lett., 2005, 59, 3986-3989.

20 H. K. Park, J. K. Yoon and K. Kim, Langmuir, 2006, 22, 1626-1629.

21 K. Kim, H. B. Lee, H. K. Park and K. S. Shin, J. Colloid Interface Sci., 2008, 318, 195-201.

22 C. Yuen, W. Zheng and Z. W. Huang, Proceeding of SPIE Plasmonics in Biology and Medicine, 2008, 6869, 86905.

23 T. Murphy, H. Schmidt and H. D. Kronfeldt, Proceeding of SPIE, 1997, 3107, 281-287.

24 J. Zhang, J. Liu, S. Wang, P. Zhan, Z. Wang and N. Ming, $A d v$. Funct. Mater., 2004, 14, 1089-1096.

25 T. Murphy, H. Schmidt and H. D. Kronfeldt, Appl. Phys. B: Lasers Opt., 1999, 69, 147-150.

26 H. Schmidt, N. BichHa, J. Pfannkuche, H. Amann, H. D. Kronfeldt and G. Kowalewska, Mar. Pollut. Bull., 2004, 49, 229-234.

27 O. Péron, E. Rinnert, M. Lehaitre, P. Crassous and C. Compère, Talanta, 2009, 79, 199-204.

28 O. Péron, E. Rinnert, M. Lehaitre, F. Colas and C. Compère, Proceeding of SPIE Defense, Security \& Sensing, 2009, 7312 73120D1-10.

29 O. Péron, E. Rinnert, M. Lehaitre, F. Colas and C. Compère, Appl. Spectrosc., 2010, 64, DOI: 10.1366/000370210792973505.
30 W. B. Lacy, L. G. Olson and J. M. Harris, Anal. Chem., 1999, 71, 2564-2570.

31 K. E. Shafer-Peltier, C. L. Haynes, M. T. Glucksberg and R. P. Van Duyne, J. Am. Chem. Soc., 2003, 125, 588-593.

32 A. Lorén, J. Engelbrektsson, C. Eliasson, M. Josefson, J. Abrahamsson, M. Johansson and K. Abrahamsson, Anal. Chem., 2004, 76, 7391-7395.

33 R. Stosch, A. Henrion, D. Schiel and B. Güttler, Anal. Chem., 2005, 77, 7386-7392.

34 A. März, K. R. Ackermann, D. Malsch, T. Bocklitz, T. Henkel and J. Popp, J. Biophotonics, 2009, 2, 232-242.

35 G. Frens, Nature, Phys. Sci., 1973, 241, 20-22.

$36 \mathrm{M}$. Hu, S. Noda, T. Okubo, Y. Yamaguchi and H. Komiyama, Appl. Surf. Sci., 2001, 181, 307-316.

37 O. Seitz, M. M. Chehimi, E. Cabet-Deliry, S. Truong, N. Felidj, C. Perruchot, V. Greaves and J. F. Watts, Colloids Surf., A, 2003, 218, 225-230.

$38 \mathrm{G}$. Socrates, Infrared and Raman characteristic group frequencies, John Wiley \& Sons Ltd, 2001, pp. 300-301.

39 L. A. Gribov, O. I. Kondratov and A. V. Kotov, Zhurnal Proikladnoi Spektrskopii, 1972, 17, 1074-1079.

40 L. Stolberg, J. Lipkowski and D. E. Irish, J. Electroanal. Chem., 1991, 300, 563-584.

41 A. M. Michaels, M. Nirmal and L. E. Brus, J. Am. Chem. Soc., 1999, 121, 9932-9939.

42 E. C. Le Ru, M. Dalley and P. G. Etchegoin, Curr. Appl. Phys., 2006, 6, 411-414.

43 D. J. Anderson and M. Moskovits, J. Phys. Chem., 2006, 100, 1372213727.

44 I. Langmuir, J. Am. Chem. Soc., 1918, 40, 1361-1403.

45 H. Freundlich, Z. Phys. Chem., 1907, 57A, 385-470. 